\title{
One Case of Diagnosis and Treatment for a Cat with Lower
}

\section{Urinary Tract Syndrome}

\author{
Chunhui XU, Yong TAO* \\ Jiangsu Agri-animal Husbandry Vocational College
}

*Corresponding Author: Ph. D. Yong TAO, professor, engaged in animal science research, Taizhou 225300, China

\begin{abstract}
: the clinical manifestations of urinary system syndrome in cats are frequent urination, painful urination, dysuria, hematuria and abnormalities in the process of urination. In this paper, we diagnosed and analyzed the past medical history; clinical symptoms, laboratory and imaging examination of a male cat with typical lower urinary tract syndrome, and analyzed the causes of the disease and recorded the diagnosis and treatment process.
\end{abstract}

Keywords: lower urinary tract; syndrome; crystalluria; symptoms; diagnosis and treatment

The lower urinary tract syndrome of cats is a general term for the bladder and urethra diseases of cats, which can be divided into two types: blocking and non-blocking [1]. Blocking lower urinary tract syndrome is caused by protein-like substances and mineral crystals blocking the urethra. The clinical symptoms are frequent licking of external genitalia, frequent urination, painful urination, body temperature decrease, vomiting, hypotension and even anuria. If the anuria lasts too long ( $24 \mathrm{~h}$ ), it will lead to acute death; Non-blocking lower urinary tract syndrome in cats is mainly caused by bladder and urethra inflammations, tumor or abnormal anatomical structure of urethra. The clinical symptoms are frequent urination, painful urination, frequent licking of external genitalia and dribbling [2]. The incidence rate of this disease is high, which is mainly related to the shortage of drinking water and the change of $\mathrm{pH}$ value of urine. If the dietary magnesium and fiber content is high, it will also promote the occurrence of this disease. Due to the narrow urethra of male cats, at the distal end of the bulbourethral gland, it is easy to form crystals and then eventually block the urethra. In general, a large crystal stone is formed at a certain position of the urethra and penis to block the urethra; the urethra of female cats is short and elastic, and the urethral passage is wide, so it is not easy to form blockage, so male cats are more likely to develop this disease than female cats. At present, the main methods of diagnosis are clinical, laboratory, B-mode ultrasound and X-ray examinations. The author experienced a case of lower urinary tract syndrome of the cat, and now we shared the diagnosis and treatment for your reference.

\section{INTRODUCTION ABOUT THE DISEASE}

Siamese, male, 5-year-old, not sterilized, and the immunity is complete. The cat has been suffering from mental depression. In the past two days, it has no appetite, did not like to drink water, often licks its external genitals, and frequently ran to the litter box, showing defecation posture. However, there is no large cat litters in the litter box, only scattered and different-size cat litters, and sometimes there are bloody cat litters. 


\section{Clinical Symptom EXamination}

The anal temperature is $39.4^{\circ} \mathrm{C}$. It is depressed in spirit, and the abdomen is bulging and slightly shaking. Palpate the abdomen to see the the tension of the cat. Sometimes, the abdomen of cats without urethral obstruction will also have pain when being pressed, but the posterior position of the abdomen of cats with urethral obstruction is extremely sensitive, and the bladder is filled and expanded, and generally, we can feel that as soon as we touch it. When touching an expanded bladder, we should be careful, because the pressure inside the bladder increases, which is likely to cause damage to the bladder wall.

\section{LABORATORY EXAMINATION}

Use blankets to wrap and fix yje cat and let it only show its forelimbs to prevent it from struggling or even biting. Collect blood, and then do routine blood test, biochemical test and blood ion test. The abnormal results of routine blood test and biochemical examination can reflect the length of time of urethral obstruction, and it may show no obvious symptoms, or severe azotemia (serum creatinine $>11 \mathrm{mg} / \mathrm{dL}$ ), hyperkalemia and metabolic acidosis. There is no significant difference between the severe renal dysfunction caused by urethral obstruction and the clinical examination results of other types of acute failure.

\subsection{Routine blood test}

Collect blood samples, use automatic blood cell analyzer to carry out blood routine examination (Table 1). The value of granalocytes was high, indicating bacterial infection, and the values of red blood cells, hemoglobin and hematocrit were high, indicating dehydration. Other indexes were in the normal reference range.

Table1. Blood routine examination results of the sick cat

\begin{tabular}{|l|l|l|l|}
\hline \multicolumn{1}{|c|}{ Items } & Results & Reference values & Tips \\
\hline White blood cell (WBC) & $19.0 \times 10^{9} / \mathrm{L}$ & $5.5 \sim 19.5$ & \\
\hline Lymphocyte (Lymph) & $3.0 \times 10^{9} / \mathrm{L}$ & $0.8 \sim 7.0$ & \\
\hline Monocyte (Mon) & $0.5 \times 10^{9} / \mathrm{L}$ & $0.0 \sim 1.9$ & \\
\hline Granulocyte (Gran) & $15.5 \times 10^{9} / \mathrm{L}$ & $2.1 \sim 15.0$ & $\mathrm{H}$ \\
\hline Red blood cell (RBC) & $10.33 \times 10^{12} / \mathrm{L}$ & $4.60 \sim 10.00$ & $\mathrm{H}$ \\
\hline Hemoglobin (HGB) & $161 \mathrm{~g} / \mathrm{L}$ & $93 \sim 153$ & $\mathrm{H}$ \\
\hline Hematocrit (HCT) & $51.6 \%$ & $28.0 \sim 49.0$ & $\mathrm{H}$ \\
\hline Mean corpuscular volume (MCV) & $50.0 \mathrm{fL}$ & $39.0 \sim 52.0$ & \\
\hline Mean corpuscular hemoglobin (MCH) & $16.7 \mathrm{pg}$ & $13.0 \sim 21.0$ & \\
\hline $\begin{array}{l}\text { Mean corpuscular hemoglobin concentration } \\
\text { (MCHC) }\end{array}$ & $347 \mathrm{~g} / \mathrm{L}$ & $300 \sim 380$ & \\
\hline Platelet count (PLT) & & & \\
\hline Mean platelet volume (MPV) & $9.9 \mathrm{fL}$ & $5.0 \sim 11.8$ & \\
\hline
\end{tabular}

\subsection{X-ray Examination}

Through X-ray examination, no obvious stone was found, but the bladder was found to be filled (Figure 1). 


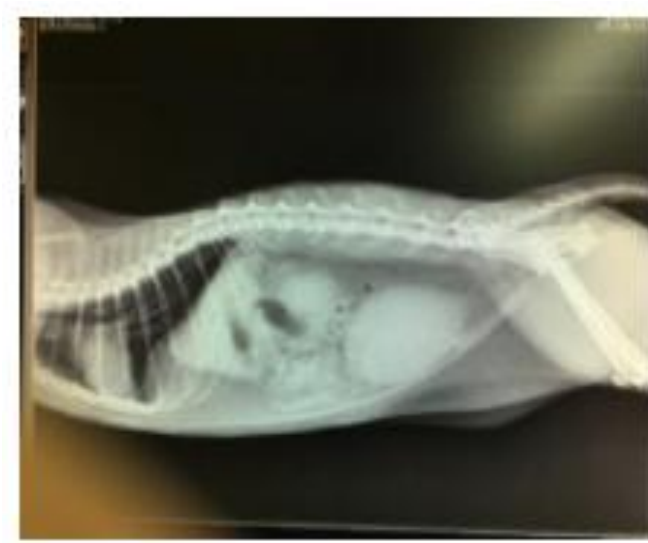

Fig.1 Through X-ray ion, the bladder was found to be filled

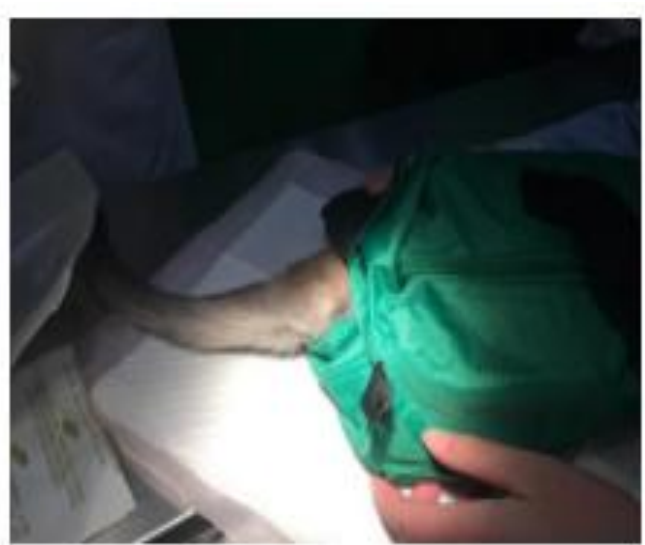

Fig. 2 Cat fixing and catheterizatio

\subsection{Blood Biochemical Examination}

Biochemical examination and results of Japan arkray biochemical analyzer. The biochemical examination for the cat with lower urinary tract syndrome mainly included urea nitrogen, creatinine, aspartate transaminase, phosphorus and lactate dehydrogenase. The results showed that the liver function and renal function were normal.

\section{DiAgNOSIS}

In the examination, we found no obvious stones, but the bladder of the cat was abnormally enlarged, the urine volume was very small and the urination was difficult, which indicated that the urethra was obstructed, but the obstruction was incomplete obstruction. Through clinical symptoms, clinical examination and laboratory examination, we preliminarily diagnosed that the cat had lower urinary tract syndrome, and the examination results were urethral orifice obstruction and bacterial infection.

\section{Cause of Disease}

The main causes of the disease are related to nutrition, pathogen infection, insufficient exercise and insufficient water drinking. (1) Nutrition and diet. Generally speaking, the nutritional structure of cats is relatively special because its diet structure is very single. If the cat is fed a high-protein diet for a long time, it is easy to cause imbalance of calcium and magnesium in the body, and excessive fiber content in dry food will promote the formation of feces, which will increase the concentration of urine, promote the formation of crystals, and then cause urinary stones and inflammation. If we feed the cat a diet that acidify urine and feed the cat urinary tract acidifiers for a long time, it will cause calcium oxalate stones, which will then block the urethra. (2) Pathogen invasion. Escherichia coli, Staphylococcus aureus, Streptococcus and Feline calicivirus are closely related to the disease. Some other diseases, such as trauma, and bladder, urethral and nerve diseases, including hemangioma, prostate cancer, fibroma, urethral spasm, bladder paralysis, etc., can lead to the decline of the cat's body resistance, which may eventually lead to the invasion of exogenous microorganisms, which may lead to the occurrence of stone disease. (3) Lack of exercise and lack of water. Fat cats generally lack exercise and rarely drink water, so its urine is less and accumulated, and when the urine can not be discharged in time, the accumulated urine will be acidified or alkalized. When the $\mathrm{pH}$ value of the cat's urine is higher than 7 , it will promote the precipitation of magnesium ammonium phosphate in large quantities, resulting in crystallization in the urine. Some sterilized cats are more anxious, which may cause the disease, and its external stress and other factors may also cause the disease. 


\section{Treatment}

\subsection{Catheterization}

Material preparation: two pairs of sterilized gloves, $500 \mathrm{ml}$ sterilized normal saline, sterilized lubricant and one catheter.

Operation method: connect the cat to the ventilator and anesthesia machine, and let it lie on its back and fix it. Use $0.1 \%$ Bromogeramine solution to clean and disinfect it, and then let the assistant to pull back the penis sheath, grab the penis and pull it out slowly. Then spray Chlorhexidine around the urethra to clean it. Apply lubricant on the end edge of sterilized catheter. Pay attention not to let the lubricant block the catheter. Insert it from the urethral orifice of penis and slowly push it into the bladder, and if there is any foreign body, inject $37^{\circ} \mathrm{C}$ saline into the catheter to try to flush the crystal stones in the urethra until it is inserted into the bladder (see Fig. 2). Take out about $150 \mathrm{ml}$ urine, the color changed from dark red to light red, and then suture the catheter to fix it (the catheter is left for 2 days), and then put on a collar to prevent the dog form being licked and bitten by itself.

\subsection{Fluid Therapy}

In order to adjust the symptoms of various ions, body fluids and electrolyte acid-base balance caused by urethral obstruction, we used fluid therapy for dogs.

Intravenous injection: the first group of drugs: $25 \mathrm{ml} 0.9 \%$ sodium chloride injection $+0.25 \mathrm{~g}$ ceftriaxone. The second group of drugs: $50 \mathrm{ml}$ compound sodium chloride injection + ATP + COA + VC $2 \mathrm{ml}+2 \mathrm{ml} \mathrm{VB6}+0.5 \mathrm{ml}$ ranitidine $+0.5 \mathrm{ml} 10 \%$ potassium chloride. The third group of drugs: $20 \mathrm{ml} \mathrm{0.9 \%} \mathrm{sodium} \mathrm{chloride} \mathrm{injection}+5 \mathrm{mg}$ Omeprazole. The fourth group of drugs: $20 \mathrm{ml} 0.9 \%$ sodium chloride injection+ $0.5 \mathrm{ml}$ lipoic acid.

Intramuscular injection: $0.6 \mathrm{ml}$ norfloxacin.

Oral drugs: Intestine Betterment, 1 bag/time, 1 time/day; Cystaid, 1 tablet/time, 2 times/day.

Continuous infusion for four days. On the second day, each indicator gradually improved; on the third and fourth days, the indicators returned to normal, urine became clear, and the cat could urinate autonomously, and its appetite returned to normal. It mainly completely recovered and discharged from hospital. Then we went on with two days of intramuscular injections for anti-inflammatory treatment. We suggested the cat owner should try to feed prescription food and let the cat drink more water.

\section{ANALYSIS AND DiSCUSSION}

For this disease, in the diagnosis process, the key is to determine whether there is urethral obstruction. When urethral obstruction occurs, palpate the bladder, and it is full, sometimes sand-like crystals appear at the external genital orifice. There is no sand mass or only a small amount of small sand mass in cat litter [4]; as for non-blocking urethral obstruction, when we touch the bladder of a cat, the bladder is soft and not filled, and sometimes when we squeeze it hard, bloody urine will flow out [5]. Through hematological examination, we can determine whether there is secondary, postrenal azotemia and whether the renal function is damaged [6]; the purpose of urine examination is to determine the acidity and alkalinity of urine and whether there are magnesium ammonium phosphate and calcium oxalate crystals in urine. Generally, there is no crystal urine in acidic urine, and at the same time, if there is no bacterial infection; we mostly consider it as spontaneous bladder. X-ray and B-type ultrasound examinations can rule out bladder stones, bladder tumor and urethral malformation [8]. 
In the treatment, we should pay attention to the following aspects: A. the correct use of urinary catheter. When urethral obstruction occurs, we can use a catheter to guide the urine out. If the bladder is full, we should puncture it first, and then conduct catheterization, and then wash the bladder repeatedly to guide the crystals in the bladder out of the body; Do not directly use the catheter to catheterize. We can connect the back end of the appropriate indwelling needle with a syringe filled with $20 \mathrm{ml}$ normal saline, and then flush the urethral orifice, and only after the crystals or blockages are washed away, can catheterization be conducted, and otherwise, it will aggravate inflammatory edema of the urethra and cause catheterization failure. When the urethra is blocked for more than 24 hours, we recommend indwelling the catheter. After solving the problem of urethral obstruction, we need to check whether there is postrenal azotemia, acidosis and hyperkalemia due to urethral obstruction. B. Use urinary tract acidifier reasonably. Only when the $\mathrm{pH}$ value of urine is alkaline, can we use urinary acidifier, because long-term use of it will cause calcium oxalate crystal formation, and this stone is insoluble. C. When there is bacterial infection, we suggest to do bacterial culture and drug sensitivity test. We must collect urine by puncturing the bladder, so as to prevent the result deviation caused by artificial pollution of urine. D. Spontaneous cystitis will be relieved within 1 week, but some will recur. The main content of conservative treatment is to give the antidepressant amitriptyline and bladder mucosa protectant. E. For the problem of urethral obstruction which can not be solved by conservative treatment, we can consider to carry out operation to deal with the problem of urethral stricture.

In the process of feeding, we need to feed scientifically and match foods reasonably, feed more wet food, and let the cat drink more water and exercise more. We should avoid frequent replacement of cat litter and cat food, which can reduce the incidence rate of cats. When the cat has frequent urination, hematuria, restlessness, loss of appetite and vomiting, or repeatedly squats on cat litter, or when there are other symptoms, we should timely send it to the pet hospital for examination, otherwise it is easy to delay the disease treatment and cause serious renal damage.

\section{ACKNOWLEDGMENT}

This work is supported by "National Development and Reform Commission of China's Bio-breeding Capacity Building Project" (20150299); "Jiangsu Modern Agricultural Industrial Technology System Construction Project"(JATS2018243).

\section{REFERENCES}

[1] Meng Peixia, Li Liyang and Deng Ganzhen. Diagnosis and treatment for lower urinary tract syndrome in male cats $[\mathrm{J}]$. Modern animal husbandry and veterinary, 2016 (04): 38-43.

[2] Guo Jianying, Han Wencai. A survey of research on urinary system syndrome in cats [J]. Heilongjiang animal science and veterinary medicine, 2014 (1): 42-44.

[3] Wu Haitao, Li Yanyan, Zhou Honglei, et al. One case of diagnosis and treatment of a cat with lower urinary tract syndrome [J]. Livestock and Poultry Industry, 2017 (11): 78-79.

[4] Zhao Li. Clinical diagnosis and treatment for lower urinary tract diseases in cats [D]. Northwest A \& F University, 2014.

[5] Tian Jianjun, Chen Yizhou, Huang Yaqi, et al. One case of cinical diagnosis and treatment of lower urinary tract syndrome and secondary bladder urine leakage in a cat [J]. Guangdong animal husbandry and veterinary science and technology, 2013,38 (5): 40-42.

[6] Liu Xu, Zhao Li. Diagnosis and treatment of lower urinary tract syndrome in cats [J]. Veterinary Guide, 2016 (7): 65-66.

[7] Zhang Wenquan. Surgical treatment of lower urinary tract syndrome in cats [J]. Fujian animal husbandry 
and veterinary medicine $[\mathrm{J}], 2014$ (6): 73-74.

[8] Pei Zhihua, Wang Kai. Clinical diagnosis and treatment of lower urinary system diseases in cats [J]. Heilongjiang animal science and veterinary medicine, 2012 (6): 106-107.

Citation: Yong TAO \& Chunhui XU (2020). "One Case of Diagnosis and Treatment for a Cat with Lower Urinary Tract Syndrome". International Journal of Medicinal Plants and Natural Products (IJMPNP), 6(4), pp.20-25. https://doi.org/ 10.20431/2454-7999.0604003

Copyright: () 2020 Authors. This is an open-access article distributed under the terms of the Creative Commons Attribution License, which permits unrestricted use, distribution, and reproduction in any medium, provided the original author and source are credited. 